\title{
The Study of Various Phenomena behind the Soil Deformation
}

\author{
Rengaswamy ARIVAZHAGAN*, Krishnamurthy PREMALATHA
}

\begin{abstract}
The nature puts no restriction and allows particles to behave freely and randomly. The soil particles deform when they are subjected to the loading, following a unique pattern. When the shear stress breaks down the original structure of a particulate mass during loading to a flow structure, steady state conditions occur. The soil deformation follows the Poisson process since the deformation follows the exponential order. The inter particle contact at the soil grains follows the concepts of simple friction. These revelations about soil behaviour paved way for a new concept called dynamic system based soil mechanics. In this model, the first order nonlinear differential equations are formed. The DSSM parameters for sand-silt mixtures under different drainage conditions decrease with increase in confining pressure. It is concluded that the applicability of this model varies with the gradation and grain size characteristics and sand silt mixtures.
\end{abstract}

Keywords: exponential decay; interaction; sand; silt; triaxial test

\section{INTRODUCTION}

The continuous movement and reorientation of soil particles during shearing results in a steady state condition where the original structure of the soil is completely lost and it deforms continuously with constant shear and normal stress, volume and velocity. Steady state condition of a soil mass explicitly differs from critical state in two aspects as described by Joseph [3]. One, the deformation of the soil is at constant velocity in steady state as against critical state, where a Pseudo velocity exists. Secondly, the soil grains in steady state are in a flow structure with continuously occurring shear strains which is not in the case of critical state. In order to understand the complex behaviour of soil, the steady state and critical state concepts have been extensively used and dealt separately in modelling the soil behaviour. Desai [2], introduced the "Disturbed state concept", which used both critical state and steady state of soil in modelling the soil behaviour. The strength and deformation behaviour of soil is understood well by steady state and critical state lines as per theory of soil dynamics. Dynamical systems in science generally are often defined by complex and chaotic processes [7]. Nevertheless, the dynamical strength and deformation process of soil can be understood in a simpler way through physical processes, for soil is a natural material which obeys the laws of nature. This approach was presented by Joseph [3] through his dynamic system based soil mechanics model for modelling the soil behaviour. The present work is an attempt to use this model for understanding the behaviour of sand silt mixtures. The concept of DSSM is as shown in Fig. 1.

The deformation of soil mass has a unique pattern, mainly it depends upon the grain sizes, loading conditions, drainage conditions, etc. The interaction of shear stress and effective normal stress during the soil deformation is referred to as the Dynamical system by Paul G Joseph [4]. In the dynamical soil-shear system, shear stress destroys the soil, and the effective normal stress pressurizes the soil. In an attempt to describe the soil behaviour through physical processes, a new approach was proposed based on Poisson process and Lotka-Volterra model. The interaction of soil particles follows the laws and simple friction and the deformation is a Poisson process.

\section{SOIL DEFORMATION AS A POISSON PROCESS}

Particles transfer forces through their contacts with other particles. The changing in load causes the particles to re-orient from their initial position because of the change in the inter particle force. This re-orientation occurs till the particles attain steady state flow structure.

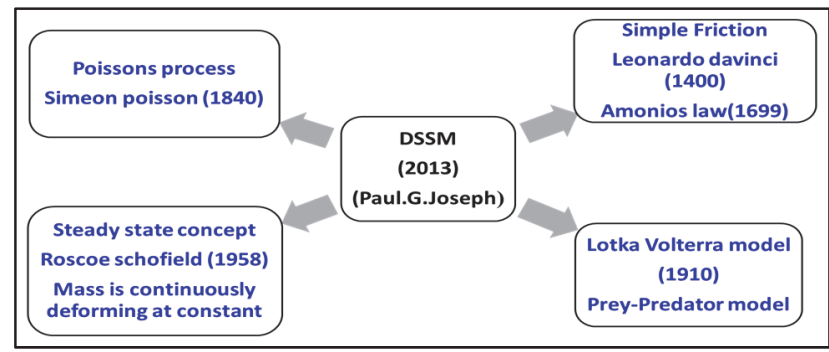

Figure 1 Concept of Dynamic System Based Soil Mechanics (DSSM)

As particles move into the steady state, the number of particles available for re-orientation under conditions of ongoing shear strain applications decreases with shear strain. The physical basis of soil deformation is a micro structure process of particle re-orientation with a particle moving into the flow structure at the same random shear strain. This is exactly like the case of radioactive decay. This decay process is an experimental process and is expressed by:

$$
N=N o \exp (-\lambda \gamma)
$$

where:

No - Initial number of particles that are not in the steadystate position,

$N$ - Remaining number of particles that are not in the steady-state at shear strain $\gamma$,

$\lambda$ - The rate at which particles move into the steady state.

\section{FORMULATION OF NONLINEAR DIFFERENTIAL EQUATIONS}

The Lotka-volterra model (1910) states how the predators and prey interact dynamically, and also this model equations also known as predators-prey equations, this was given as 
$\frac{\mathrm{d} x}{\mathrm{~d} t}=\alpha x-\beta x y$

$\frac{\mathrm{d} y}{\mathrm{~d} t}=\gamma y+\delta x y$

where:

$x$ - the number of prey (eg: rabbits),

$y$ - The number of predator (eg: foxes),

$\alpha, \beta, \gamma, \delta$ - non-negative parameters.

The above Eq. (2) express that the rate of change of prey's population is given by its own growth rate minus the rate at which it is preyed upon and Eq. (3) expresses that the rate of change at the predator's population depends upon the rate at which is consumes prey, minus its intrinsic death rate.

This understanding of predator-prey model helps to model a soil deformation as a dynamical system. That is shear stress trying to destroy the soil structure as a predator and the confining stress that seems to preserve soil structure as prey. During the deformation, the particles moves to new orientation as the steady state flow structure, the most of the soil particles will affect both shear stress ' $q$ ' and effective normal stress $\bar{p}$.

\section{SOIL DEFORMATION FOLLOWS SIMPLE FRICTION}

During the soil deformation, most of the soil particles will affect both $q$ and $\bar{p}$, some particles affect only $q$ or only $\bar{p}$.

$N \bar{p}$ and $N q$ are the number of particles that affect $\bar{p}$ and $q$ respectively.

$J p$ and $J q$ are the corresponding interparticle contact area per unit sample area, they are non-dimensional.

$\lambda p$ and $\lambda q$ are the rate of decrease of $J p$ and $J q$ respectively.

The Poisson process and the predator-prey equations combined to form a differential equation and expressed as:

$$
\begin{aligned}
& \frac{\mathrm{d} q}{\mathrm{~d} \gamma}=J q[\bar{p} \tan \alpha q \exp (-\lambda p \gamma)-q \exp (-\lambda q \gamma)] \\
& \frac{\mathrm{d} \bar{p}}{\mathrm{~d} \gamma}=J p[\bar{p} \tan \alpha q \exp (-\lambda p \gamma)-q \exp (-\lambda q \gamma)]
\end{aligned}
$$

In the above Eq. (4) and Eq. (5), $J q \tan \alpha_{q}$ and $J p \tan \alpha_{p}$ are replaced by $A$ and $L$ respectively.

$J q$ and $J p$ are replaced by $J$ and $K$ respectively.

$\lambda p$ and $\lambda q$ are replaced by $B$ and $D$ respectively. Then the Eq. (4) can be modified as equations as follows

The physical behavior described alone expressed mathematically as:

$$
\begin{aligned}
& \frac{\mathrm{d} q}{\mathrm{~d} \gamma}=\bar{p} A \exp (-B \gamma)-q J \exp (-D \gamma) \\
& \frac{\mathrm{d} \bar{p}}{\mathrm{~d} \gamma}=\bar{p} A \exp (-B \gamma)-q J \exp (-D \gamma)
\end{aligned}
$$

where: $q$ - shear stress; $\bar{p}$ - Effective normal stress; $A, J, L$ $\& K$ are the proportional constants and stand for the initial measures of the resistance offered by the soil structure to $p$ and $q$ respectively. $B \& D$ are the exponential rates at which these proportional constants decay with strain to zero.

\section{EVALUATION OF PARAMETERS}

$A, L, J$ and $K$ are the proportional constants and stand for the initial measures of the resistance offered by the soil structure to $p$ and $q$ respectively

$B$ and $D$ are the exponential rates at which these proportional constants decay with strain to zero.

The Eq. (6) and Eq. (7) can be solved through standard forth order Runge-Kutta numerical integration scheme, but prior to that the model parameters:

$\bar{V}=[A, I, J, K, B, D]$

needs to be computed. First, based on the experimental values of $(\bar{p}, q)$. The values of $p=\left(\sigma_{1}+\sigma_{3}\right) / 2$ and

$q=\left(\sigma_{1}-\sigma_{3}\right) / 2$ are calculated from the triaxial test results.

$\frac{\mathrm{d} q}{\mathrm{~d} \gamma}=q_{i+1}-q_{i} / \Delta \gamma$

$\frac{\mathrm{d} \bar{p}}{\mathrm{~d} \gamma}=\bar{p}_{i+1}-\bar{p}_{i} / \Delta \gamma$

$\mathrm{d} q / \mathrm{d} \gamma$ and $\mathrm{d} \bar{p} / \mathrm{d} \gamma$ are calculated by forward difference scheme. To find $\bar{V}$, the Eq. (6) and Eq. (7) can be solved to get six independent variables, which is clearly an inconsistent system. Hence apply an optimization solver with a given initial conditions for $\bar{V}$, by:

$\overline{V_{0}}=\left[A_{0}, I_{0}, J_{0}, K_{0}, B_{0}, D_{0}\right]$

Thus, the objective function required to solve can be written as $\operatorname{Min}[\|\mathrm{d} q / \mathrm{d} q-\bar{p} A \exp (-\beta \gamma)+q J \exp (-D \gamma)\|+\|$ $\mathrm{d} p / \mathrm{d} \gamma-\bar{p} L \exp (-\beta \gamma)+q K \exp (-D \gamma) \|]$ where $\|-\|$ represents the norm of a vector. The above optimization problem has been solved using MATLAB software along with $\overline{V_{0}}$. The convergence /accuracy of the solution $\bar{V}$, is heavily dependent on the given initial condition $\overline{V_{0}}$.

Thus to verify the solution $\bar{V}$, Eq. (6) and Eq. (7) was solved using the Runge - Kutta fourth order method and compared the solutions $(\bar{p}, \mathrm{q})$ with the experimental values of $(\bar{p}, q)$. If the results are not found satisfactory, iterate the values till the satisfactory fitting of the curves.

\section{EXPERIMENTAL PROGRAM}

A series of static triaxial tests were conducted on samples of sand, silt and sand-silt mixtures under 
consolidated drained conditions and consolidated undrained conditions at various confining pressures of 100 $\mathrm{kPa}, 200 \mathrm{kPa}$ and $300 \mathrm{kPa}$. A density of $1.47-1.57 \mathrm{~g} / \mathrm{cc}$ was maintained for all the tests. All the tests were conducted on loose state. The procedures used during the triaxial testing program are based on IS 2720-Part III, 1993. Mild moist tamping method was adopted for the sample preparations and the sample size was height $76 \mathrm{~mm}$ and diameter $38 \mathrm{~mm}$.

\section{RESULTS AND DISCUSSION}

Model parameters of DSSM were calculated for all the samples of sand-silt mixtures. Selected curves for which the best fit was obtained is presented in Fig. 2 and Fig. 3 which show the normalized stress strain curve plotted between $q / \sigma_{c}$ and $p / \sigma_{c}$ with axial strain $\epsilon(\%)$ for S60M40 and S100 for the confining pressure of $300 \mathrm{kPa}$ under Consolidated Undrained condition.

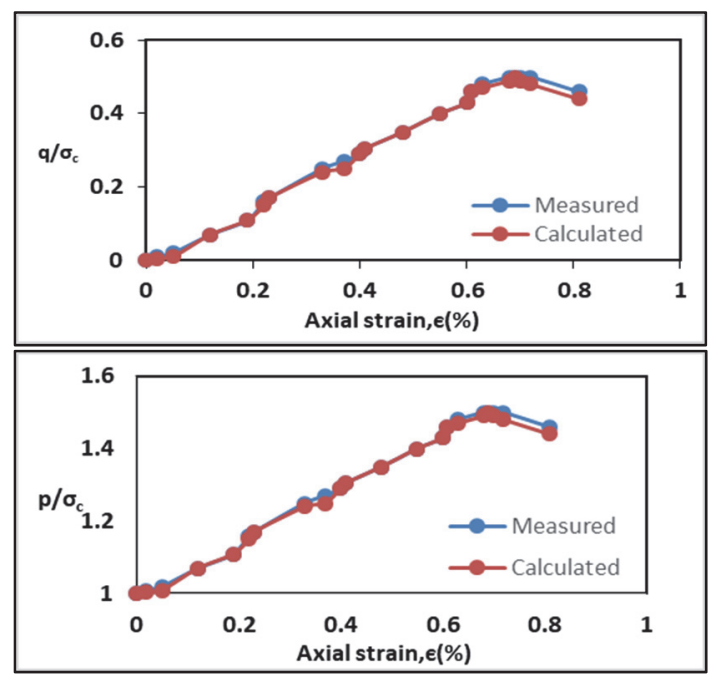

Figure 2 Measured against calculated normal Stress-Strain curve for S60M40 at $300 \mathrm{kPa}$ for $\mathrm{CU}$ condition

Fig. 4 and Fig. 5 show the normalized stress strain curve plotted between $q / \sigma_{c}$ and $p / \sigma_{c}$ with shear strain $\gamma$ for S70M30 and M100 for the confining pressure of $300 \mathrm{kPa}$ under consolidated drained condition.

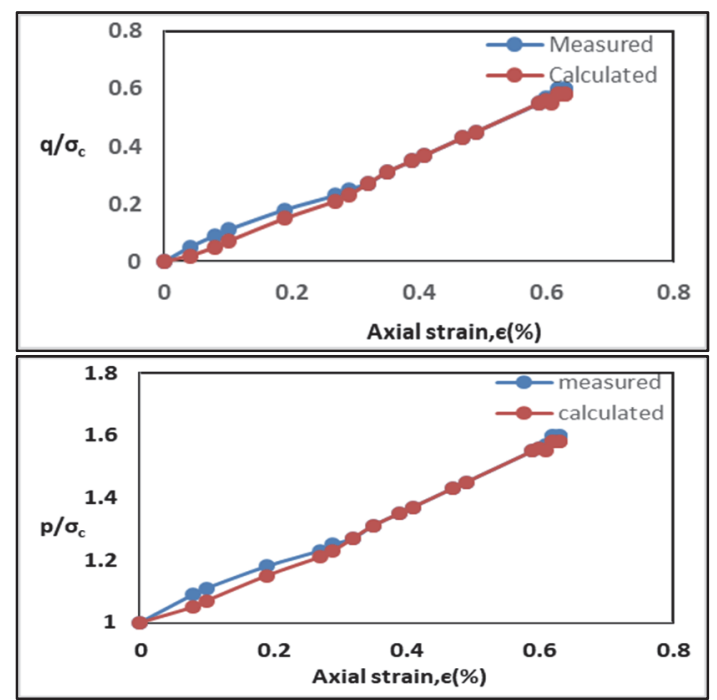

Figure 3 Measured against calculated normal stress strain curve for S100 at $300 \mathrm{kPa}$ for CU condition

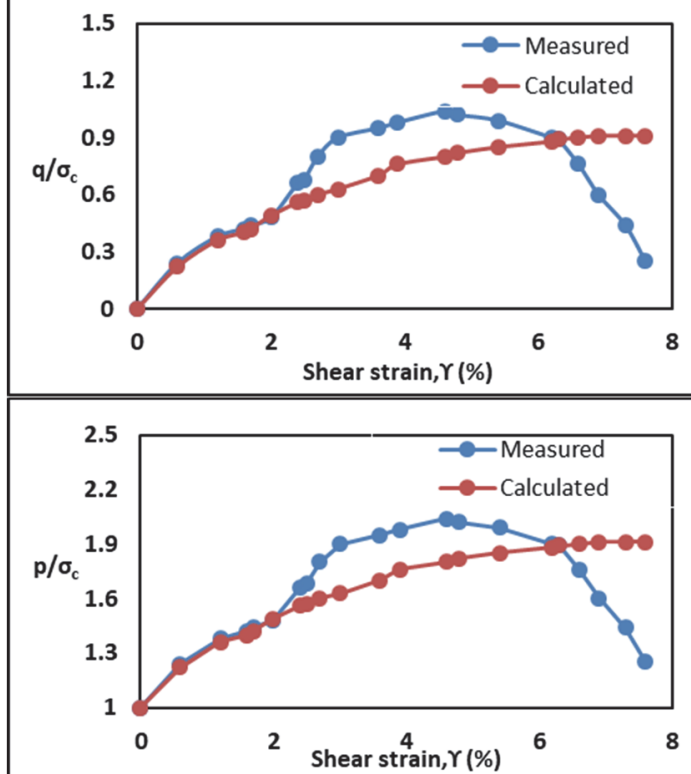

Figure 4 Measured against calculated normal stress strain curve for S70M30 at $300 \mathrm{kPa}$ for $\mathrm{CD}$ condition

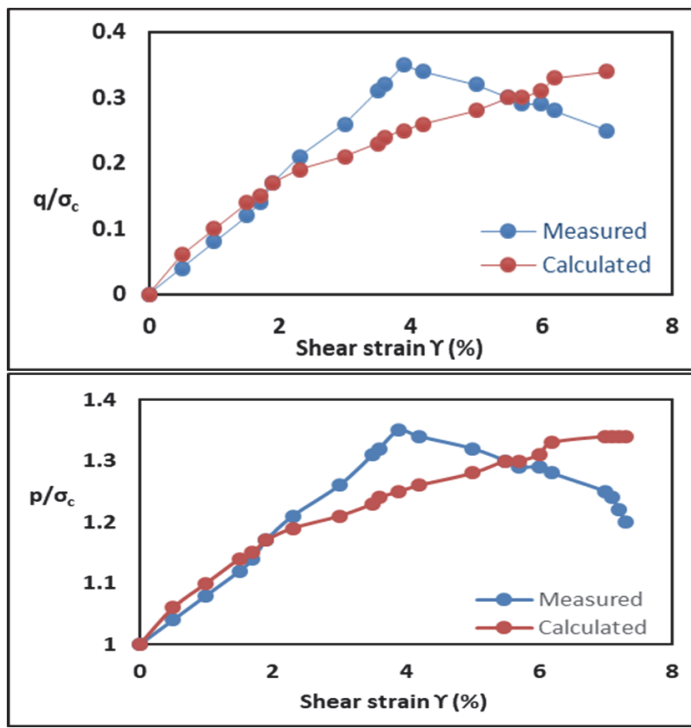

Figure 5 Measured against calculated normal stress strain curve for M100 at $300 \mathrm{kPa}$ for $\mathrm{CD}$ condition

The model parameters are evaluated from the above curves using Matlab software and listed in Tab. 1 and Tab. 2 . The variation of model parameters with confining pressures is presented in Fig. (6) and Fig. (7). From Tab. 1 and Tab. 2, and Fig. 6 and Fig. 7, it is observed that the model parameter values of $A, J, K, L, B$ and $D$ decrease with increase in confining pressure. Joseph [3] reported that this dynamical system based model shows typical fit to data from Shehan [6], Arulmoli, et. al. [1] and Sharpiro [5] for drained compression and extension tests on clay, sand and silt. The influence of confining pressure on the model parameters reported by the authors is the same as the influence observed in the present study. 


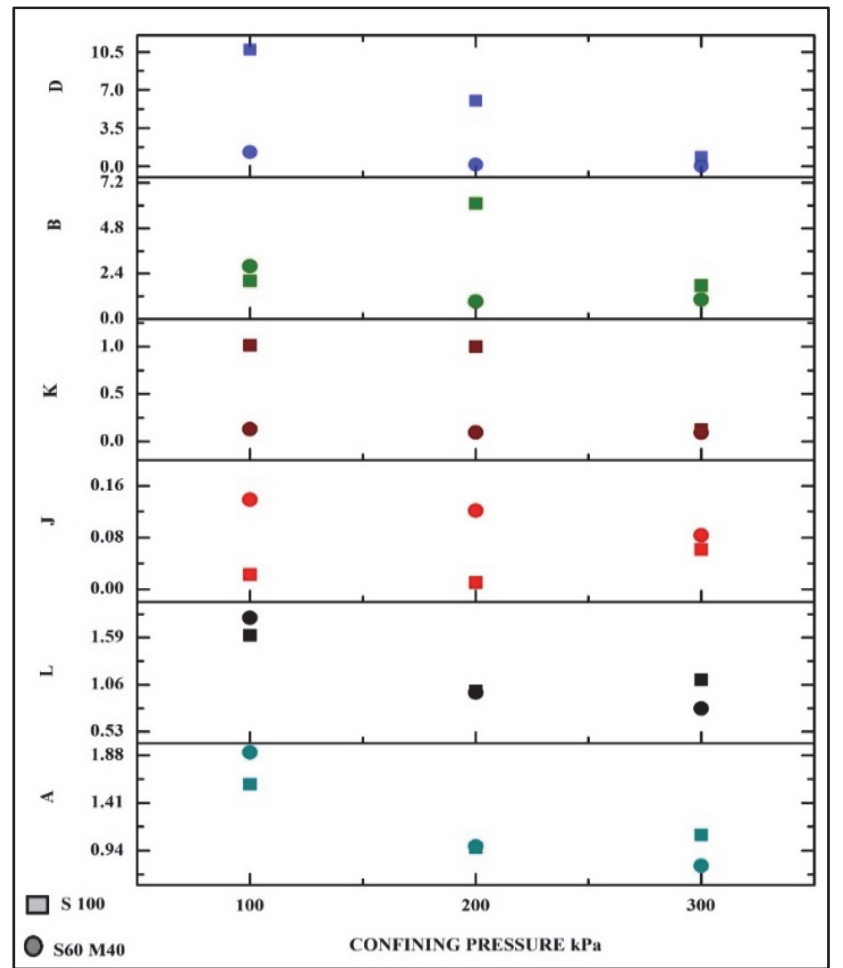

Figure 6 Variation of Model parameters with confining pressures at consolidated undrained condition

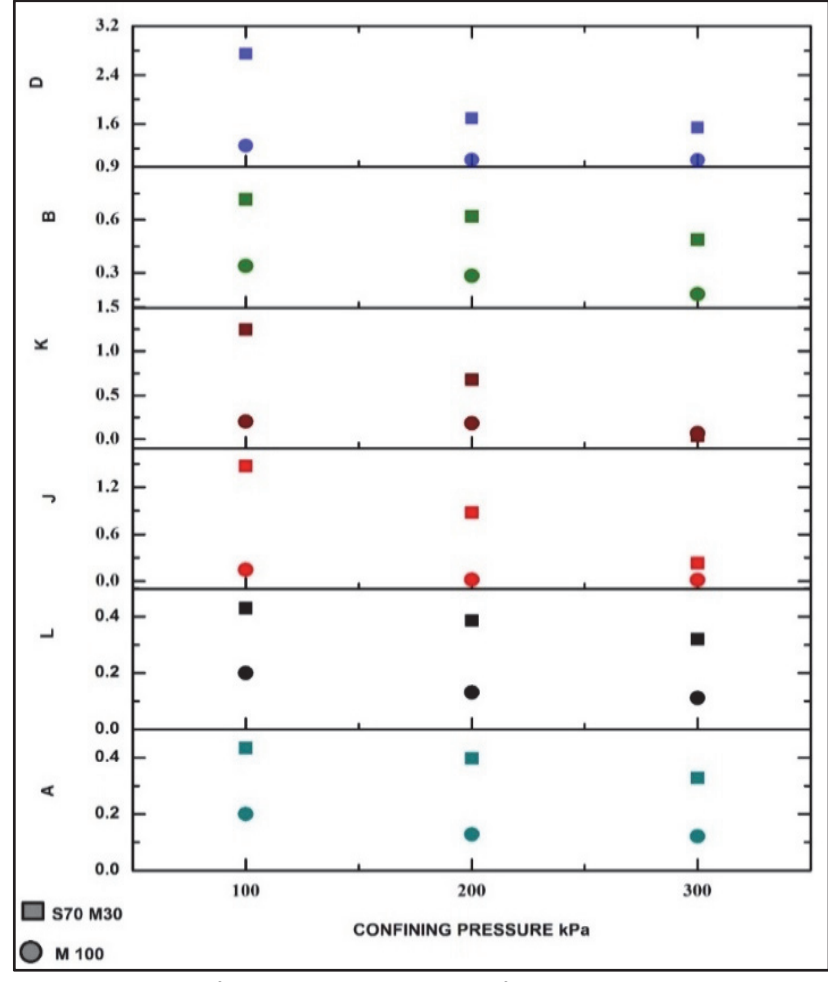

Figure 7 Variation of Model parameters with confining pressures at consolidated drained condition

Table 1 Model Parameters of Sand and Sand-Silt Mixture at Consolidated Undrained condition

\begin{tabular}{|c|c|c|c|c|c|c|c|}
\hline \multirow[b]{2}{*}{ Sample } & \multirow[b]{2}{*}{$\begin{array}{c}\text { Confining Pressure } \\
\qquad / \mathrm{kPa}\end{array}$} & \multicolumn{6}{|c|}{ Model Parameters } \\
\hline & & $A$ & $J$ & $L$ & $K$ & $B$ & $D$ \\
\hline \multirow{3}{*}{$\mathrm{S} 100$} & 100 & 1,5928 & 1,617 & 0,0225 & 1,0128 & 2,0231 & 10,66 \\
\hline & 200 & 0,965 & 0,987 & 0,01 & 1,001 & 6,099 & 6,01 \\
\hline & 300 & 1,0963 & 1,1152 & 0,0612 & 0,1193 & 1,7695 & 0,8513 \\
\hline \multirow{3}{*}{ S60M40 } & 100 & 1,9099 & 1,8137 & 0,1384 & 0,1285 & 2,795 & 1,3085 \\
\hline & 200 & 0,9794 & 0,9693 & 0,1216 & 0,0916 & 0,9281 & 0,1424 \\
\hline & 300 & 0,7876 & 0,7893 & 0,0833 & 0,0886 & 1,0333 & 0,0217 \\
\hline
\end{tabular}

Table 2 Model Parameters of Sand and Sand-Silt Mixture at Consolidated Drained condition

\begin{tabular}{|c|c|c|c|c|c|c|c|}
\hline \multirow[b]{2}{*}{ Sample } & \multirow[b]{2}{*}{$\begin{array}{c}\text { Confining Pressure } \\
\qquad / \mathrm{kPa}\end{array}$} & \multicolumn{6}{|c|}{ Model Parameters } \\
\hline & & $A$ & $J$ & $L$ & $K$ & $B$ & $D$ \\
\hline \multirow{3}{*}{ S70M30 } & 100 & 0,4334 & 0,4334 & 0,4299 & 1,4721 & 1,248 & 0,7162 \\
\hline & 200 & 0,3974 & 0,3974 & 0,3866 & 0,8801 & 0,6759 & 0,6194 \\
\hline & 300 & 0,3281 & 0,3281 & 0,3199 & 0,2311 & 0,0387 & 0,4866 \\
\hline \multirow{3}{*}{ M100 } & 100 & 0,1997 & 0,1997 & 0,2001 & 0,146 & 0,2021 & 0,3387 \\
\hline & 200 & 0,1279 & 0,1279 & 0,1313 & 0,0193 & 0,1828 & 0,282 \\
\hline & 300 & 0,1207 & 0,1207 & 0,1118 & 0,0188 & 0,0704 & 0,1782 \\
\hline
\end{tabular}

\section{CONCLUSION}

This paper made an experimental programme to validate the dynamical systems based approach to soil shear. The test results show that the rates of change of shear stress q, effective normal stress $\bar{p}$ are proportional to the applied shear and effective normal stresses. The proportional values decay exponentially as the soil structure changes, which follows the Poisson process, in which particles move at random shear strains into the final structure. This model made a bridge between the soil deformations to a powerful mathematical framework. The inter particle contact area $J q$ and $J p$, the secant friction angles $\alpha_{q}$ and $\alpha_{p}$ are all replaced by the parameters $A, J, K$, $L, B$ and $D$ in the mathematical framework. This makes all the parameters signify that the model parameters have a known physical basis.

The DSSM parameters $A, J, K, L, B$ and $D$ for sand silt mixtures under consolidated drained and Consolidated Undrained condition decrease with increase in confining pressure. It is concluded that the applicability of this model varies with the gradation and grain size characteristics of sand silt mixtures. The DSSM model best fitted with the measured and calculated data for the sand and sand-silt mixtures under consolidated undrained conditions rather than the consolidated drained conditions. In consolidated drained conditions, at lower level of shear strain that is up 
to $2 \%$, the measured and calculated data closely fit with each other.

\section{Acknowledgements}

The authors would like to thank Dr. Mahadevan, Riyanka Irene Sureshraj and Pradeep Kumar. Sincerest gratitude to the Secretary, Dr.K.S. Babai and Principal Dr.P.K.Suresh, Meenakshi Sundararajan Engineering College, Kodambakkam, Chennai-24, for their support to do all the research activities.

\section{REFERENCES}

[1] Arulmoli, K., Muraleetharan, K. K., Hossain, M. M., \& Fruth, L. S. (1992). Verification Verification of Liquefaction Analyses by Centrifuge Studies Laboratory Testing Program Soil Data Report. The Earth Technology Corporation, Irvine, CA, USA, report to the National Science Foundation.

[2] Chandrakant, S. D. (2015). Disturbed state concept as unified constitutive modeling approach. Journal of Rock Mechanics and Geotechnical Engineering.

[3] Joseph, P. G. (2017). Dynamical systems-Based Soil Mechanics, CRC Press Taylor \& French Group. https://doi.org/10.1201/9781315193137

[4] Joseph, P. G. \& Graham-Eagle, J. (2013). Strain-Rate Effects in Shear High-lighted by a Dynamical systems Model. Int. J. Geomech., 14(4), $\quad 04014015, \quad 1-8$ https://doi.org/10.1061/(ASCE)GM.1943-5622.0000360

[5] Shapiro. S. (2000). The effects of non-plastic fines on the three-dimensional behaviour of Sand. M.S.Thesis, Clarkston University, New York.

[6] Sheahan, T. C. (1991). An Experimental Study of Time Dependant Undrained Shear and Behaviour of Resedimented Clay Using Automated Stress Path Triaxial Equipment. ScD Thesis, Massachusetts Institute of Technology, Cambridge, MA, USA.

[7] Strogatz, S. H. (1994). Nonlinear Dynamics and Chaos. Perseus Publishing Company, New York.

Contact information:

Rengaswamy ARIVAZHAGAN, Assistant Professor, PhD, FIGS

(Corresponding author)

Meenakshi Sundararajan Engineering College,

A3, R.E. ApartmentsKrishnapuram Street, Choolaimedu,

Chennai-94

E-mail: Arivu000@gmail.com

Krishnamurthy PREMALATHA, Professor, PhD, MIGS

College of Engineering Guindy,

No.19, Second cross street,

Rathinamani Nagar, Perungudi,

Chennai-96

E-mail:kvpremalatha@yahoo.com 